\title{
Derivation and Solution of Harmonic Riccati Equations via Contraction Mapping Theorem
}

\author{
Jun ZHOU*
}

\begin{abstract}
By the contraction mapping theorem and the harmonic Lyapunov equation theory, sufficient existence conditions of what we call the harmonic Riccati equations in finite-dimensional linear continuous-time periodic systems are explicated. Properties of the harmonic Riccati equations are also examined. Different from the Hamiltonian analysis, the approach reveals some analytic properties of periodic solutions of periodic matrix Riccati equations. An iterative algorithm is suggested for solving periodic matrix Riccati equations, which only involves algebraic Lyapunov equations and Fourier coefficients of periodically time-varying matrices.
\end{abstract}

Key Words: Toeplitz operator, Riccati equation, periodic system, contraction mapping, iterative solution

\section{Introduction}

In linear quadratic regulation (LQR) problems of finitedimensional linear continuous-time periodic (FDLCP) models ${ }^{3), 7), 16)}$, researchers must frequently tackle Riccati equations with periodically time-varying coefficients $\left.\left.\left.\left.\left.{ }^{1}\right), 14\right), 15\right), 17\right), 19\right)$. To deal with such Riccati equations, various theoretic and numeric tools are proposed; numeric solution of differential equations, quasilinearization and Hamiltonian theory are well-adopted ones. The first method is effective in the numeric sense ${ }^{9)}$ in general; the second is a Newton-type algorithm and leads a necessary and sufficient existence condition of periodic solutions ${ }^{1}{ }^{2}{ }^{24}$, while the third not only provides numeric solutions but also is indispensable in theoretical analysis $\left.\left.{ }^{1)}, 17\right), 19\right)$. Fourier analysis is also powerful in tackling the periodic matrix Riccati equations, but generally it is used as an approximation tool ${ }^{16)}$. Recently Fourier analysis is considered ${ }^{18)}$ in establishing a class of operator-valued Riccati equations in FDLCP setting. The motivation behind this attempt ${ }^{18)}$ is straightforward; that is, if the periodic matrix Riccati equation can be re-cast as an operator-valued one that is similar in form to that of LTI continuous-time systems ${ }^{10)}$, then it is expected that results about LQR problems of LTI continuous-time systems can be extended to FDLCP cases. However, the arguments in 18) are not persuasive and consist mainly of intuitive observations since

* Department of Electrical Engineering, Faculty of Engineering, Kyoto University, Kyotodaigaku-Katsura, Nishikyo-ku, Kyoto

(Received August 30, 2007)

(Revised December 25, 2007) some important convergence issues related to differentiation and product of periodically time-varying functions in periodic Riccati differential equations are not scrutinized when Fourier analysis is employed.

In the paper, we attack existence of what we call the harmonic Riccati equations through the contraction mapping theorem ${ }^{8), 13)}$ and the harmonic Lyapunov equation ${ }^{21)}$. This is motivated by the facts that the solution of a harmonic Lyapunov equation ${ }^{21), 22)}$ is always available, and that convergence of a contraction iteration can be guaranteed. To show the existence in this way, neither do we assume that the periodic matrix Riccati equation concerned has a periodic solution, nor do we express the periodic solution of the periodic matrix Riccati equation via the Hamiltonian theory (thus get rid of finite escape times and initial solutions problems $\left.\left.{ }^{1)}, 8\right), 17\right)$. This approach is essentially different from the Hamiltonian theory and sheds new light on LQR problems in FDLCP setting and periodic matrix Riccati equations. Indeed, we do not simply stop after verifying the existence of the harmonic Riccati equations. The solution of the harmonic Riccati equation is further connected with periodic solutions of the periodic matrix Riccati equation. This leads an iterative algorithm for asymptotic computations of periodic solutions of the periodic matrix Riccati equations.

Notations. The spaces $l_{2}, C^{n}[0, h], L_{2}[0, h]$ and $L_{\infty}[0, h]$ are standard ${ }^{13)}$. A function matrix $F(t) \in L_{2}[0, h]$ means that each element of $F(t)$ is $h$-periodic and belongs to $L_{2}[0, h]$ when it is restricted to $[0, h] . \mathcal{Z}$ is the set of all integers. In the paper, both real and complex FDLCP systems are considered. To validate the Toeplitz transformation on periodic functions (see 20),21) for the details), we defined the following sets. $L_{\mathrm{PCD}}[0, h]$ is the 
set of all piecewise continuous functions that are differentiable almost everywhere in $[0, h] ; L_{\mathrm{CD}}[0, h]$ is the set of all continuous functions and differentiable a.e. in $[0, h]$, while $L_{\mathrm{CAC}}[0, h]$ is that of all continuous functions whose Fourier series are absolutely convergent.

\section{Preliminaries to FDLCP Systems}

\section{Consider the FDLCP system}

$$
G: \dot{x}=A(t) x+B(t) u
$$

where $A(t)$ and $B(t)$ are $n \times n$ and $n \times m h$-periodically time-varying matrices, respectively. By the Floquet theorem ${ }^{3), 11)}$, if $A(t)$ is locally integrable, the transition matrix $\Phi(t, 0)$ of (1) has a Floquet factorization $\Phi(t, 0)=$ $P(t, 0) e^{Q t}$, where $P(t, 0)$ is absolutely continuous in $t$, nonsingular and $h$-periodic in $t$ and $t_{0}$, and $Q$ is constant. The system is asymptotically stable if and only if all the eigenvalues of $Q$ have negative real parts. Major analytic features of Floquet factorizations and their numeric algorithms can be found in 23).

The Toeplitz transformation. Expand $X(t) \in L_{2}[0, h]$ to its Fourier series $\sum_{m=-\infty}^{+\infty} X_{m} e^{j m \omega_{h} t}$ with $\omega_{h}=2 \pi / h$. The Toeplitz transformation on $X(t)$ maps $X(t)$ into an infinite-dimensional Toeplitz operator ${ }^{18)}$

$$
\mathcal{T}\{X(t)\}=\left[\begin{array}{ccccc}
\ddots & \vdots & \vdots & \vdots & \cdot \\
\cdots & X_{0} & X_{-1} & X_{-2} & \cdots \\
\cdots & X_{1} & X_{0} & X_{-1} & \cdots \\
\cdots & X_{2} & X_{1} & X_{0} & \cdots \\
. & \vdots & \vdots & \vdots & \ddots
\end{array}\right]=\underline{X}
$$

where $X(t)$ is called the defining function of $\underline{X}^{5 \text {, p. 565) }}$. Here we further write $\underline{A}=\mathcal{T}\{A(t)\}, \underline{P}=\mathcal{T}\{P(t, 0)\}$, $\underline{Q}=\mathcal{T}\{Q\}=\operatorname{diag}[\cdots, Q, Q, Q, \cdots]$ and

$$
\underline{E}(j 0)=\operatorname{diag}\left[\cdots,-j 2 \omega_{h} I,-j \omega_{h} I, 0, j \omega_{h} I, j 2 \omega_{h} I, \cdots\right]
$$

Also we define $l_{E}=\left\{\underline{x} \in l_{2}: \underline{E}(j 0) \underline{x} \in l_{2}\right\}$, which is a proper subset of $l_{2}$ and dense in $l_{2}{ }^{20}$ ).

Lemma 1. In the system (1), let $A(t) \in L_{\mathrm{PCD}}[0, h]$. Then, $\underline{P}$ is bounded on $l_{2}$ and invertible in $l_{2}$ and $l_{E}$ with $\underline{P}^{-1}$ being bounded on $l_{2}$. The unbounded operators $\underline{P}(\underline{E}(j 0)-\underline{Q}) \underline{P}^{-1}$ and $\underline{E}(j 0)-\underline{A}$ are densely defined on $l_{2}$ (or equivalently, well-defined on $l_{E} \subset l_{2}$ ) and it holds true on $l_{E} \subset l_{2}$ that $\underline{P}(\underline{E}(j 0)-\underline{Q}) \underline{P}^{-1}=\underline{E}(j 0)-\underline{A}$.

By slightly modifying the arguments in 21), Lemma 2 can be verified, which describes properties of the harmonic Lyapunov equations. The harmonic Lyapunov equation plays a key role in deriving the harmonic Riccati equations in the FDLCP setting.
Lemma 2. In the system (1), assume that $A(t) \in$ $L_{\mathrm{PCD}}[0, h]$ is asymptotically stable. For any Toeplitz operator $\underline{W}$ on $l_{2}$, there exists a unique bounded solution $\underline{V}$ satisfying

$$
(\underline{A}-\underline{E}(j 0))^{*} \underline{V}+\underline{V}(\underline{A}-\underline{E}(j 0))=-\underline{W}
$$

which is called the harmonic Lyapunov equation of the system (1) and holds on $l_{E} \subset l_{2}$. Moreover, the unique solution $\underline{V}$ of (2) can be expressed as follows.

$$
\underline{V}=\underline{P}^{-*} \int_{0}^{\infty} \underline{e}(Q, \tau)^{*} \underline{P^{*}} \underline{W} \underline{P} \underline{e}(Q, \tau) d \tau \underline{P}^{-1}
$$

Here $\underline{e}(Q, t)=\operatorname{diag}\left[\cdots, e^{\left(Q+j \omega_{h} I\right) t}, e^{Q t}, e^{\left(Q-j \omega_{h} I\right) t}, \cdots\right]$ and $\underline{P}^{-*}=\left[\underline{P}^{-1}\right]^{*}$.

Note that $L_{\infty}[0, h] \subset L_{2}[0, h]$. Hence, the following set is well-defined.

$$
\mathcal{X}=\left\{\underline{X}=\mathcal{T}\{X(t)\}: X(t) \in L_{\infty}[0, h]\right\}
$$

By Corollary 2.2 of 5 , p. 567), if $\underline{X} \in \mathcal{X}$, then $\|\underline{X}\|_{l_{2} / l_{2}}=$ $\operatorname{essup}_{t \in[0, h]}\|X(t)\|$. Since the Fourier series for any $X(t) \in L_{\infty}[0, h]$ is unique, it follows that $\mathcal{T}: L_{\infty}[0, h] \rightarrow$ $\mathcal{X}$ is one-to-one. Now we state the following lemma. A proof is given in Appendix.

Lemma 3. Let $\mathcal{X}$ be defined in (4). Then, the metric space $\left(\mathcal{X},\|\cdot\|_{l_{2} / l_{2}}\right)$ is a Banach space.

\section{Main Results}

In this section, we first construct an iterative algorithm based on Lemma 2, and then the harmonic Riccati equation is derived by making this algorithm into a contraction mapping, whose properties are also examined.

\section{1 Derivation of Harmonic Riccati Equations}

Theorem 1. Suppose in the system (1) that $A(t) \in$ $L_{\mathrm{PCD}}[0, h]$ is asymptotically stable and $B(t) \in L_{\infty}[0, h]$. Let the cost function matrices $U(t), R(t)$ belong to $L_{\infty}[0, h]$ and there exists a number $\gamma>0$ such that the set $\{t:|\operatorname{det}[R(t)]|<\gamma\}$ has measure zero. If there exists a number $\mu>0$ such that the inverse of $R(t)$ satisfies

$$
\begin{aligned}
& \operatorname{ess} \sup _{t \in[0, h]}\left\|R^{-1}(t)\right\| \\
< & \min \left\{\frac{1}{2 K_{2}\left(K_{1}+\mu\right)}, \frac{\mu}{K_{2}\left(K_{1}+\mu\right)^{2}}\right\}
\end{aligned}
$$

then the harmonic Riccati equation defined on $l_{E} \subset l_{2}$

$$
\begin{aligned}
& (\underline{A}-\underline{E}(j 0))^{*} \underline{X}+\underline{X}(\underline{A}-\underline{E}(j 0)) \\
- & \underline{X} \underline{B} \underline{R}^{-1} \underline{B}^{*} \underline{X}+\underline{U}=0
\end{aligned}
$$

has a unique solution $\underline{X} \in \mathcal{X}_{K_{1}+\mu}$. Moreover, the defining function $X(t)$ of $\underline{X}$, i.e., $\mathcal{T}\{X(t)\}=\underline{X}$, belongs to $L_{\infty}[0, h]$. Here $K_{1}$ and $K_{2}$ are defined in (11), and the 
subset $\mathcal{X}_{K_{1}+\mu}$ of $\mathcal{X}$ is defined in (12).

Proof The assumption that the set $\{t: \operatorname{det}|[R(t)]|<\gamma\}$ has measure zero for sufficiently small $\gamma>0$ means that $R(t)$ may be singular at some isolated points at most. From this fact, Theorem 2.4 and its proof of 5, p.569) say that $\underline{R}=\mathcal{T}\{R(t)\}$ is invertible and $\underline{R}^{-1}$ is bounded on $l_{2}$.

Now let us consider the following infinite-dimensional operator-valued iterative algorithm.

$$
\left\{\begin{array}{c}
(\underline{A}-\underline{E}(j 0))^{*} \underline{X}_{k+1}+\underline{X}_{k+1}(\underline{A}-\underline{E}(j 0)) \\
=-\underline{U}+\underline{L}_{k} \\
\underline{L}_{k}=\underline{X}_{k} \underline{B}^{-1} \underline{B}^{*} \underline{X}_{k}
\end{array}\right.
$$

where $k=0,1,2, \cdots$. By Lemma 2 , for each $k, \underline{X}_{k+1}$ is bounded on $l_{2}$ and given by

$$
\underline{P}^{-*} \int_{0}^{\infty} \underline{e}(Q, \tau)^{*} \underline{P}^{*}\left(\underline{U}-\underline{L}_{k}\right) \underline{P} \underline{e}(Q, \tau) d \tau \underline{P}^{-1}
$$

To establish the harmonic Riccati equations, we show that there exists $\underline{X} \in \mathcal{X}$ such that (7) holds when both $\underline{X}_{k+1}$ and $\underline{X}_{k}$ are replaced simultaneously by $\underline{X}$. To this end, we notice that all the eigenvalues of $Q$ have negative real parts. It follows ${ }^{3)}$ that for some $K_{Q}>0$ and $\alpha>0, \operatorname{Re}\{\lambda(Q)\}<-\alpha$ and $\left\|e^{Q t}\right\| \leq K_{Q} e^{-\alpha t}, \forall t \geq 0$. The diagonal structure of $\underline{e}(Q, t)$ leads that $\forall t \geq 0$,

$$
\|\underline{e}(Q, t)\|_{l_{2} / l_{2}}=\sup _{i \in \mathcal{Z}}\left\|e^{\left(Q+j i \omega_{h} I\right) t}\right\| \leq K_{Q} e^{-\alpha t}
$$

Using (9) into (8), simple manipulations give that

$$
\begin{aligned}
& \left\|\underline{X}_{k+1}\right\|_{l_{2} / l_{2}} \\
\leq & \left\|\underline{P}^{-1}\right\|_{l_{2} / l_{2}}^{2}\|\underline{P}\|_{l_{2} / l_{2}}^{2} \int_{0}^{\infty} K_{Q}^{2} e^{-2 \alpha \tau} d \tau \\
& \cdot\left(\|\underline{U}\|_{l_{2} / l_{2}}+\left\|\underline{X}_{k} \underline{B} \underline{R}^{-1} \underline{B}^{*} \underline{X}_{k}\right\|_{l_{2} / l_{2}}\right) \\
\leq & \left\|\underline{P}^{-1}\right\|_{l_{2} / l_{2}}^{2}\|\underline{P}\|_{l_{2} / l_{2}}^{2} \frac{K_{Q}^{2}}{2 \alpha} \\
& \cdot\left(\|\underline{U}\|_{l_{2} / l_{2}}+\left\|\underline{X}_{k}\right\|_{l_{2} / l_{2}}^{2}\left\|\underline{B}_{l_{l_{2} / l_{2}}}^{2}\right\| \underline{R}^{-1} \|_{l_{2} / l_{2}}\right) \\
= & K_{1}+K_{2}\left\|\underline{R}^{-1}\right\|_{l_{2} / l_{2}}\left\|\underline{X}_{k}\right\|_{l_{2} / l_{2}}^{2}
\end{aligned}
$$

where

$$
\left\{\begin{array}{l}
K_{1}=\left\|\underline{P}^{-1}\right\|_{l_{2} / l_{2}}^{2}\|\underline{P}\|_{l_{2} / l_{2}}^{2}\|\underline{U}\|_{l_{2} / l_{2}} \frac{K_{Q}^{2}}{2 \alpha} \\
K_{2}=\left\|\underline{P}^{-1}\right\|_{l_{2} / l_{2}}^{2}\|\underline{P}\|_{l_{2} / l_{2}}^{2}\|\underline{B}\|_{l_{2} / l_{2}}^{2} \frac{K_{Q}^{2}}{2 \alpha}
\end{array}\right.
$$

Lemma 1 says that $K_{1}$ and $K_{2}$ are finite.

Now we assume that for $\mu>0,\left\|\underline{X}_{k}\right\|_{l_{2} / l_{2}} \leq K_{1}+\mu$. Then $\left\|\underline{X}_{k+1}\right\|_{l_{2} / l_{2}} \leq K_{1}+\mu$ if $\left\|\underline{R}^{-1}\right\|_{l_{2} / l_{2}} \leq \frac{\mu}{K_{2}\left(K_{1}+\mu\right)^{2}}$. Obviously, for a fixed $\mu>0$, such a cost function $R(t)$ always exists. To facilitate our statements, define the closed subset $\mathcal{X}_{K_{1}+\mu}$ of the Banach space $\mathcal{X}$ as

$$
\mathcal{X}_{K_{1}+\mu}=\left\{\underline{X} \in \mathcal{X}:\|\underline{X}\|_{l_{2} / l_{2}} \leq K_{1}+\mu\right\}
$$

Hence, the above arguments indicate that the mapping $\underline{X}_{k} \mapsto \underline{X}_{k+1}$ of (8) reduces to a mapping on $\mathcal{X}_{K_{1}+\mu}$ if $R(t)$ satisfies $\operatorname{ess} \sup _{t \in[0, h]}\left\|R^{-1}(t)\right\|=\left\|\underline{R}^{-1}\right\|_{l_{2} / l_{2}} \leq$ $\frac{\mu}{K_{2}\left(K_{1}+\mu\right)^{2}}$. Here Corollary 2.2 of 5, p.567) was used.

Next let us consider the mapping of (8) only on $\mathcal{X}_{K_{1}+\mu}$ and define

$$
\begin{aligned}
& \underline{X}_{k+1}-\underline{\tilde{X}}_{k+1}=-\underline{P}^{-*} \int_{0}^{\infty} \underline{e}(Q, \tau)^{*} \underline{P}^{*} \\
& \cdot\left(\underline{X}_{k} \underline{B} \underline{R}^{-1} \underline{B}^{*} \underline{X}_{k}-\underline{\tilde{X}}_{k} \underline{B}^{-1} \underline{B}^{*} \underline{\tilde{X}}_{k}\right) \\
& \cdot \underline{P} \underline{e}(Q, \tau) d \tau \underline{P}^{-1}
\end{aligned}
$$

Manipulations similar to those around (10) yield

$$
\begin{aligned}
\left\|\underline{X}_{k+1}-\underline{\tilde{X}}_{k+1}\right\|_{l_{2} / l_{2}} \leq & 2\left(K_{1}+\mu\right) K_{2}\left\|\underline{R}^{-1}\right\|_{l_{2} / l_{2}} \\
& \cdot\left\|\underline{X}_{k}-\underline{\tilde{X}}_{k}\right\|_{l_{2} / l_{2}}
\end{aligned}
$$

Now choose $R(t)$ such that $2\left(K_{1}+\mu\right) K_{2}\left\|\underline{R}^{-1}\right\|_{l_{2} / l_{2}}<1$; or equivalently, let $\left\|\underline{R}^{-1}\right\|_{l_{2} / l_{2}}<\frac{1}{2 K_{2}\left(K_{1}+\mu\right)}$. Then, by definition ${ }^{13}$, p. ${ }^{125)}$, the mapping in (8) becomes a contraction mapping from $\mathcal{X}_{K_{1}+\mu}$ to $\mathcal{X}_{K_{1}+\mu}$ if $\left\|\underline{R}^{-1}\right\|_{l_{2} / l_{2}}<$ $\min \left\{\frac{1}{2 K_{2}\left(K_{1}+\mu\right)}, \frac{\mu}{K_{2}\left(K_{1}+\mu\right)^{2}}\right\}$. From this, the contraction mapping theorem ${ }^{8, \text { p.77), 13, p.126) }}$ and Lemma 3 lead that there exists a unique operator $\underline{X} \in \mathcal{X}_{K_{1}+\mu}$ such that (7) holds for $\underline{X}_{k+1}=\underline{X}_{k}=\underline{X}$, which is nothing but (6).

Remark 1. Theorem 1 provides sufficient existence conditions for existence of (6). It is established without the assumption that the periodic matrix Riccati equation

$$
\begin{aligned}
-\dot{X}(t)= & A^{T}(t) X(t)+X(t) A(t) \\
& -X(t) B(t) R^{-1}(t) B^{T}(t) X(t)+U(t)
\end{aligned}
$$

has a periodic solution. In 18), the harmonic Riccati equation (6) is asserted by applying the Toeplitz transformation directly to (14) under the assumption that (14) has a periodic solution. However, the arguments in 18) are not persuasive since key convergence issues related to the Toeplitz transformations on differentiations and products of periodically time-varying functions are simply neglected. In view of this, existence of the harmonic Riccati equation is proved rigorously here for the first time.

\section{2 Properties of Harmonic Riccati Equations}

Theorem 1 does not assert that the defining function $X(t)$ of the solution $\underline{X}$ of $(6)$ is a periodic solution of (14), though this is seemingly obvious and highly expected. Before drawing such a conclusion, other convergence problems must be tackled. More specifically, to connect the solution of (6) with that of (14) rigorously, we must show that the Fourier series of the derivative of $X(t)$, i.e., $\dot{X}(t)$, has 'strong' enough convergence such that the differentiation of $X(t)$ can be done on the Fourier series of $X(t)$. 
Theorem 2 below connects the solution of (6) with a periodic solution of (14). The major difference between Theorem 1 and Theorem 2 is that the assumptions on $A(t), B(t)$ and the cost functions $U(t), R(t)$ are strengthened. These strengthened assumptions will surmount the aforementioned convergence problems.

Theorem 2. Suppose in the system (1) that $A(t) \in$ $L_{\mathrm{CD}}[0, h]$ is asymptotically stable and $B(t) \in L_{\mathrm{CAC}}[0, h]$. Let the cost functions $U(t) \in L_{\mathrm{CAC}}[0, h]$ and $R^{-1}(t) \in$ $L_{\mathrm{CAC}}[0, h]$. If for a given number $\mu>0,(5)$ holds true, then the defining function $X(t)$ of the solution $\underline{X}$ of $(6)$ is continuous and continuously differentiable with respect to $t \in[0, h]$, i.e., $X(t) \in C^{1}[0, h]$, and is a periodic solution of the periodic matrix Riccati equation (14).

Proof The proof is given by modifying that of Theorem 3 of 22). By the Floquet theorem and basic properties (see Theorem 6.3 .2 of 11$)$ ) about $\Phi(t, 0)$, we have

$$
\left\{\begin{array}{l}
P(t, 0)=\Phi(t, 0) e^{-Q t} \\
\dot{P}(t, 0)=[A(t) \Phi(t, 0)-\Phi(t, 0) Q] e^{-Q t} \\
P^{-1}(t, 0)=e^{Q t} \Phi(0, t) \\
\dot{P}^{-1}(t, 0)=e^{Q t}[Q \Phi(0, t)-\Phi(0, t) A(t)]
\end{array}\right.
$$

which implies by $A(t) \in L_{\mathrm{CD}}[0, h]$ that $P^{-1}(t, 0)$ and $P(t, 0)$ belong to $C^{1}[0, h]$. Hence, Theorem 2 of 2, p. 104) tells that $P(t, 0)$ and $P^{-1}(t, 0)$ belong to $L_{\mathrm{CAC}}[0, h]$.

To see the main results, let us return to (8) and suppose that we have finished the $k$-th step of the iteration algorithm of (7) and are in a position to fix $\underline{X}_{k+1}$ by (8). Assume that in the $k$-th step we get $\underline{X}_{k}$ which has a defining function $X_{k}(t)$ belonging to $L_{\mathrm{CAC}}[0, h]$. Under these assumptions, if the Fourier coefficients sequence of $X_{k}(t)$ is denoted by $\left\{X_{i}^{(k)}\right\}_{i=-\infty}^{\infty}$, Theorem 3 of 4 , p. 155) tells us that $X_{k}(t)=\sum_{i=-\infty}^{\infty} X_{i}^{(k)} e^{j i \omega_{h} t}$ for each $t \in[0, h]$.

To simplify our expressions, we further define

$$
\begin{aligned}
M_{k}(t)= & P^{T}(t, 0)(U(t) \\
& \left.-X_{k}(t) B(t) R^{-1}(t) B^{T}(t) X_{k}(t)\right) P(t, 0)
\end{aligned}
$$

By the assumptions on $B(t), U(t), R^{-1}(t)$ and $X_{k}(t)$, we can conclude that $M_{k}(t) \in L_{\mathrm{CAC}}[0, h]$.

By denoting $\underline{M}_{k}=\mathcal{T}\left\{M_{k}(t)\right\}$, the algorithm (8) can be equivalently rewritten as follows.

$$
\begin{aligned}
\underline{X}_{k+1} & =\underline{P}^{-*} \int_{0}^{\infty} \underline{e}(Q, \tau)^{*} \underline{M}_{k} \underline{e}(Q, \tau) d \tau \underline{P}^{-1} \\
& =: \underline{P}^{-*} \underline{N}_{k} \underline{P}^{-1}
\end{aligned}
$$

where $\underline{N}_{k}$ has the obvious definition. Furthermore, we denote the defining function of $\underline{N}_{k}$ by $N_{k}(t)$ and show that $N_{k}(t)$ is continuous and continuously differentiable.

To this end, let $\left\{N_{i}^{(k)}\right\}_{i=-\infty}^{\infty}$ and $\left\{M_{i}^{(k)}\right\}_{i=-\infty}^{\infty}$ be the
Fourier sequences of $N_{k}(t)$ and $M_{k}(t)$, respectively. We show that the Fourier series $\sum_{i=-\infty}^{\infty} N_{i}^{(k)} e^{j i \omega_{h} t}$ of $N_{k}(t)$ is absolutely convergent. From (16), the block-diagonal feature of $\underline{e}(Q, \tau)$ yields

$$
N_{p}^{(k)}=\int_{0}^{\infty} e^{\left(Q+j p \omega_{h} I\right)^{*} \tau} M_{p}^{(k)} e^{Q \tau} d \tau
$$

This implies by noting $M_{k}(t) \in L_{\mathrm{CAC}}[0, h]$ that

$$
\begin{aligned}
\sum_{p=-\infty}^{\infty}\left\|N_{p}^{(k)}\right\| & \leq \int_{0}^{\infty}\left\|e^{Q \tau}\right\|^{2} d \tau \sum_{p=-\infty}^{\infty}\left\|M_{p}^{(k)}\right\| \\
& \leq \frac{K_{Q}^{2}}{2 \alpha} \sum_{p=-\infty}^{\infty}\left\|M_{p}^{(k)}\right\|<\infty
\end{aligned}
$$

Hence the Fourier series of $N_{k}(t)$ is absolute and thus uniformly convergent over $t \in[0, h]$. It follows that $N_{k}(t)$ is continuous in $t$. This, together with Theorem 3 of 4 , p. 155), implies that

$$
N_{k}(t)=\sum_{i=-\infty}^{\infty} N_{i}^{(k)} e^{j i \omega_{h} t}, \quad \forall t \in[0, h]
$$

Using this in (16) and noting that $P^{-1}(t, 0) \in L_{\mathrm{CAC}}[0, h]$, we can assert that the defining function $X_{k+1}(t)$ of $\underline{X}_{k+1}$ belongs to $L_{\mathrm{CAC}}[0, h]$ and satisfies

$$
X_{k+1}(t)=P^{-T}(t, 0) N_{k}(t) P^{-1}(t, 0)
$$

Next we show that $N_{k}(t)$ is continuously differentiable. To see this, multiplying (17) with $j p \omega_{h}$ and using the partial integral formula, we obtain that

$$
\begin{aligned}
& j p \omega_{h} N_{p}^{(k)}=-\int_{0}^{\infty} e^{Q^{*} \tau} M_{p}^{(k)} e^{Q \tau} d e^{-j p \omega_{h} \tau} \\
= & -\left.e^{Q^{*} \tau} M_{p}^{(k)} e^{Q \tau} e^{-j p \omega_{h} \tau}\right|_{0} ^{\infty} \\
& +\int_{0}^{\infty} d\left(e^{Q^{*} \tau} M_{p}^{(k)} e^{Q \tau}\right) e^{-j p \omega_{h} \tau} \\
= & M_{p}^{(k)}+Q^{*} \int_{0}^{\infty} e^{\left(Q+j p \omega_{h} I\right)^{*} \tau} M_{p}^{(k)} e^{Q \tau} d \tau \\
& +\int_{0}^{\infty} e^{\left(Q+j p \omega_{h} I\right)^{*} \tau} M_{p}^{(k)} e^{Q \tau} d \tau Q
\end{aligned}
$$

since the system is asymptotically stable. Then from (9), (21) and $M_{k}(t) \in L_{\mathrm{CAC}}[0, h]$, we have

$$
\begin{aligned}
\sum_{p=-\infty}^{\infty}\left\|j p \omega_{h} N_{p}^{(k)}\right\| \leq & \left(1+\frac{\|Q\| K_{Q}^{2}}{\alpha}\right) \\
& \cdot \sum_{p=-\infty}^{\infty}\left\|M_{p}^{(k)}\right\|<\infty
\end{aligned}
$$

Based on (19) and (22), we observe that

$$
\begin{aligned}
& \left\|\lim _{\Delta t \rightarrow 0} \frac{N_{k}(t+\Delta t)-N_{k}(t)}{\Delta t}\right\| \\
= & \left\|\sum_{i=-\infty}^{\infty} N_{i}^{(k)} \lim _{\Delta t \rightarrow 0} \frac{e^{j i \omega_{h}(t+\Delta t)}-e^{j i \omega_{h} t}}{\Delta t}\right\|
\end{aligned}
$$




$$
\begin{aligned}
& =\left\|\sum_{i=-\infty}^{\infty} j i \omega_{h} N_{i}^{(k)} e^{j i \omega_{h} t}\right\| \\
& \leq \sum_{i=-\infty}^{\infty}\left\|j i \omega_{h} N_{i}^{(k)}\right\|<\infty
\end{aligned}
$$

where the limit and the infinite summation is interchanged, which can be validated by the differentiation theorem of power series (e.g., Theorem 8.2 of 6, p. 523)) since for each fixed $i, \lim _{\Delta t \rightarrow 0}\left(e^{j i \omega_{h}(t+\Delta t)}-e^{j i \omega_{h} t}\right) / \Delta t=$ $j i \omega_{h} e^{j i \omega_{h} t}$ and $\left\{N_{i}^{(k)}\right\}_{i=-\infty}^{\infty}$ is absolutely convergent by (18). (23) says that $N_{k}(t)$ is differentiable for each $t \in[0, h]$ and the derivative of $N_{k}(t)$ is continuous in $t$ (since $\left\{j i \omega_{h} N_{i}^{(k)}\right\}_{i=-\infty}^{\infty}$ is absolutely convergent).

These arguments tell that if the initial value $X_{0}(t)$ belongs to $C^{1}[0, h]$ and satisfies $\max _{t \in[0, h]}\left\|X_{0}(t)\right\| \leq K_{1}+\mu$, then for each $k=1,2,3, \cdots, X_{k}(t) \in C^{1}[0, h]$ and $\max _{t \in[0, h]}\left\|X_{k}(t)\right\| \leq K_{1}+\mu$ by induction. Moreover,

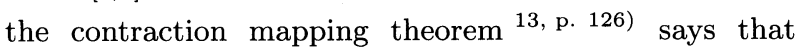
$\left\{X_{k}(t)\right\}_{k=0}^{\infty}$ is a Cauchy sequence; i.e., the defining func-

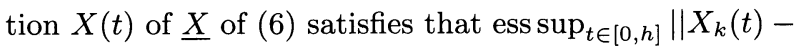
$X(t) \| \rightarrow 0$ as $k \rightarrow \infty$.

To complete the proof, it remains to show that $X(t) \in$ $C^{1}[0, h]$. We observe by (21) and (23) that

$$
\begin{aligned}
& \dot{N}_{k}(t)=\sum_{p=-\infty}^{\infty} M_{p}^{(k)} e^{j p \omega_{h} t} \\
& +\sum_{p=-\infty}^{\infty} Q^{*} \int_{0}^{\infty} e^{Q^{*} \tau} M_{p}^{(k)} e^{j p \omega_{h}(t-\tau)} e^{Q \tau} d \tau \\
& +\sum_{p=-\infty}^{\infty} \int_{0}^{\infty} e^{Q^{*} \tau} M_{p}^{(k)} e^{j p \omega_{h}(t-\tau)} e^{Q \tau} d \tau Q \\
& =M_{k}(t)+Q^{*} \int_{0}^{\infty} e^{Q^{*} \tau} M_{k}(t-\tau) e^{Q \tau} d \tau \\
& +\int_{0}^{\infty} e^{Q^{*} \tau} M_{k}(t-\tau) e^{Q \tau} d \tau Q
\end{aligned}
$$

where the integrals and summations are interchanged, which are valid by the Levi theorem (Theorem D.8.1 of 13, p.577)) since the latter two infinite summations in the first equation of (24) are absolutely convergent.

Differentiating $X_{k+1}(t)$ in (20) with respect to $t$ and using (24), we obtain after lengthy algebras that

$$
\begin{aligned}
& \left\|X_{k_{1}+1}(\cdot)-X_{k_{2}+1}(\cdot)\right\|_{*} \\
= & \max _{t \in[0, h]}\left\|X_{k_{1}+1}(t)-X_{k_{2}+1}(t)\right\| \\
& +\max _{t \in[0, h]}\left\|\dot{X}_{k_{1}+1}(t)-\dot{X}_{k_{2}+1}(t)\right\| \\
\leq & \left\{1+2 \max _{t \in[0, h]}\left\|\dot{P}^{-1}(t, 0)\right\| \max _{t \in[0 ; h]}\left\|P^{-1}(t, 0)\right\|\right. \\
& \cdot \max _{t \in[0, h]}\|P(t, 0)\|^{2} \\
& +2\left(1+\|Q\| K_{Q}^{2} / \alpha\right)\left(K_{1}+\mu\right) \max _{t \in[0, h]}\left\|P^{-1}(t, 0)\right\|^{2}
\end{aligned}
$$

$$
\begin{aligned}
& \left.\cdot \max _{t \in[0, h]}\|P(t, 0)\|^{2} \max _{t \in[0, h]}\left\|B(t) R^{-1}(t) B^{T}(t)\right\|\right\} \\
& \cdot \max _{t \in[0, h]}\left\|X_{k_{1}}(t)-X_{k_{2}}(t)\right\| \\
=: & K \max _{t \in[0, h]}\left\|X_{k_{1}}(t)-X_{k_{2}}(t)\right\|
\end{aligned}
$$

where $K<\infty$ has the obvious definition. It follows from (25) that $\left\{X_{k}(t)\right\}_{k=0}^{\infty}$ is also a Cauchy sequence in $C^{1}[0, h]$ but in the sense of the norm $\|\cdot\|_{*}$. Bearing this in mind, we notice that $\left(C^{1}[0, h],\|\cdot\|_{*}\right)$ is complete ${ }^{12, \text { p. 137) }}$. It follows that $X_{k}(t)$ must converge to $X(t)$ with $X(t)$ belonging to $C^{1}[0, h]$.

To claim that $X(t)$ is a periodic solution of (14), we mention that $\mathcal{T}\{\dot{X}(t)\}=\underline{E}(j 0) \underline{X}-\underline{X} \underline{E}(j 0)$ holds since $X(t) \in C^{1}[0, h]^{20)}$. Then, it follows from (6) that

$$
\begin{aligned}
& \mathcal{T}\left\{A^{T}(t) X(t)+X(t) A(t)+\dot{X}(t)+U(t)\right. \\
& \left.-X(t) B(t) R^{-1}(t) B(t)^{T} X(t)\right\}=0
\end{aligned}
$$

which yields (14) since the Toeplitz transformation $\mathcal{T}$ is one-to-one.

Remark 2. In Theorem 2, $X(t) \in C^{1}[0, h]$ and thus $X(t) \in L_{\mathrm{CAC}}[0, h]$ by Theorem 2 of 2 , p. 104). This is useful in forming an iterative algorithm by modifying (7) in order to solve (6). This means that an iterative algorithm is established for solving (14), which is essentially different from the Hamiltonian approach ${ }^{1}$.

\section{3 Iterative Solution of Periodic Matrix Ric- cati Equations}

Theorem 2 connects the solution of (6) with one of the periodic solutions of (14). This means that if the solution of (6) can be fixed through (7), then one periodic solution of (14) can be found.

One may suggest to square truncate the infinitedimensional matrices in (6) as was proposed in 18); i.e., to take a finite-dimensional square partial equation out of (6). This method may work and has its own value. Unfortunately, however, since the equation (6) consists of unbounded operators like $\underline{E}(j 0)$, it is nontrivial to show convergence. We avoid this convergence problem by modifying (7) as follows in the light of Lemma 1.

$$
\left\{\begin{array}{l}
(\underline{Q}-\underline{E}(j 0))^{*} \underline{\hat{X}}_{k+1}+\underline{\hat{X}}_{k+1}(\underline{Q}-\underline{E}(j 0)) \\
=-\underline{\hat{U}}+\underline{\hat{L}}_{k} \\
\underline{\hat{L}}_{k}=\underline{\hat{X}}_{k} \underline{\hat{B}} \underline{R}^{-1} \underline{\hat{B}}^{*} \underline{\hat{X}}_{k}
\end{array}\right.
$$

where $\underline{\hat{X}}_{k}=\underline{P}^{*} \underline{X} \underline{P}, \underline{\hat{B}}=\underline{P}^{-1} \underline{B}, \underline{\hat{L}}_{k}=\underline{\hat{X}}_{k} \underline{\hat{B}} \underline{R}^{-1} \underline{\hat{B}}^{*} \underline{\hat{X}}_{k}$, and $\underline{\hat{U}}=\underline{P}^{*} \underline{U} \underline{P}$. It follows that if the transition matrix of the system (1) is available, then under the assumptions of Theorem 2, (27) has a unique solution $\underline{\hat{X}}$ with a defining function belonging to $L_{\mathrm{CAC}}[0, h]$ when $\underline{\hat{X}}_{0}$ is given properly. Knowing $\underline{\hat{X}}$, the periodic solution $X(t)$ of (14) 
will be $P^{-T}(t, 0) \hat{X}(t) P^{-1}(t, 0)$, where $\hat{X}(t)$ is the defining function of $\underline{\hat{X}}$. Now we see how to fix $\underline{\hat{X}}$ by $(27)$.

Since $\hat{X}(t) \in L_{\mathrm{CAC}}[0, h]$, we can estimate it as accurate as desired if sufficiently finitely many Fourier coefficients of $\hat{X}(t)$ can be fixed. Let us determine the first $4 q+1$ Fourier coefficients of $\hat{X}(t)$; that is, $\left\{\hat{X}_{-2 q}, \cdots, \hat{X}_{-1}, \hat{X}_{0}, \hat{X}_{1}, \cdots, \hat{X}_{2 q}\right\}$. Note that $\underline{Q}-\underline{E}(j 0)$ is block-diagonal. Then,

$$
\left\{\begin{array}{c}
{\left[(\underline{Q}-\underline{E}(j 0))^{*}\right]^{(q)}\left[\underline{\hat{X}}_{k+1}\right]^{(q)}+\left[\underline{\hat{X}}_{k+1}\right]^{(q)}} \\
\cdot[\underline{Q}-\underline{E}(j 0)]^{(q)}=-[\underline{\hat{U}}]^{(q)}+\left[\underline{L}_{k}\right]^{(q)} \\
{\left[\underline{\hat{L}}_{k}\right]^{(q)}=\left[\underline{\hat{X}}_{k} \underline{\hat{B}} \underline{R}^{-1} \underline{\hat{B}}^{*} \underline{\hat{X}}_{k}\right]^{(q)}}
\end{array}\right.
$$

where $[\cdot]^{(q)}$ is the $(2 q+1) \times(2 q+1)$ sub-matrix at the center of $(\cdot)$. Since (28) is just a portion of $(27)$, no convergence problems occur. By (28), knowing $\underline{\hat{X}}_{k}$ and thus its defining function $\hat{X}_{k}(t),\left[\underline{\underline{L}}_{k}\right]^{(q)}$ can be formed by the Fourier coefficients of $\hat{X}_{k}(t) \hat{B}(t) R^{-1}(t) \hat{B}(t)^{T} \hat{X}_{k}(t)$. Using $\left[\underline{\hat{L}}_{k}\right]^{(q)}$ in $(28),\left[\underline{\hat{X}}_{k+1}\right]^{(q)}$ can be determined by solving an algebraic Lyapunov equation. Hence, an iteration for finitely many Fourier coefficients of $\hat{X}(t)$ is established.

Strictly speaking, for each $k, \hat{X}_{k}(t)$ is not the exact defining function of $\underline{\hat{X}}_{k}$ since only a sub-matrix of $\underline{\hat{X}}_{k}$ is actually computed. Fortunately, since the exact defining function of $\underline{\hat{X}}_{k}$ belongs to $L_{\mathrm{CAC}}[0, h]$ (see the proof of Theorem 2) for each $k$, the approximation can be as accurate as desired if $q$ is large enough. The contraction mapping ensures that the iteration converges even if an approximation of $\hat{X}_{k}(t)$ is used.

Now assume in the system (1) that the transition matrix has a Floquet factorization $\Phi(t, 0)=P(t, 0) e^{Q t}$ and all the assumptions of Theorem 2 are satisfied. Denote

$$
\begin{aligned}
& Q(q)= \operatorname{diag}\left[Q+j q \omega_{h} I, \cdots, Q+j \omega_{h} I, Q,\right. \\
&\left.Q-j \omega_{h} I, \cdots, Q-j q \omega_{h} I\right] \\
& X(k, q)=\left[\begin{array}{cccc}
\hat{X}_{k, 0} & \hat{X}_{k,-1} & \cdots & \hat{X}_{k,-2 q} \\
\hat{X}_{k, 1} & \hat{X}_{k, 0} & \cdots & \vdots \\
\vdots & \vdots & \ddots & \vdots \\
\hat{X}_{k, 2 q} & \cdots & \cdots & \hat{X}_{k, 0}
\end{array}\right]
\end{aligned}
$$

and $X_{k, 2 q}(t)=\sum_{i=-2 q}^{2 q} \hat{X}_{k, i} e^{j i \omega_{h} t}$. Let $\hat{U}_{i}$ and $\hat{L}_{k, i}$ be the Fourier coefficients of $P^{T}(t, 0) U(t) P(t, 0)$ and $X_{k, 2 q}(t) P^{-1}(t, 0) B(t) R^{-1}(t) B^{T}(t) P^{-T}(t, 0) X_{k, 2 q}(t)$, respectively. $U(q)$ and $L(k, q)$ are similar to $X(k, q)$ but in terms of $\left\{\hat{U}_{i}\right\}_{i=-2 q}^{2 q}$ and $\left\{\hat{L}_{k, i}\right\}_{i=-2 q}^{2 q}$, respectively. The iterative algorithm is given below when $X_{0,2 q}(t)$ is given.

Step 1. Construct $X_{k, 2 q}(t)$; Step 2. Compute the Fourier coefficients of the periodic matrix $X_{k, 2 q}(t) P^{-1}(t, 0) B(t) R^{-1}(t) B^{T}(t) P^{-T}(t, 0) X_{k, 2 q}(t)$ and form $L(k, q)$; Step 3. Solve $Q(q)^{*} X(k+1, q)+X(k+$ $1, q) Q(q)=-U(q)+L(k, q)$ for $X(k+1, q)$; Step 4. If $\|X(k+1, q)-X(k, q)\| \leq$ error tolerance, then stop and form $X_{k+1,2 q}(t)$; otherwise let $k=k+1$ and go to Step 1.

\section{Numerical Examples}

Here we solve the periodic matrix Riccati equation corresponding to the $\pi$-periodic model ${ }^{3)}$ given below by the iterative algorithm, when the cost function $U(t)$ is a $2 \times 2$ identity and $R(t)=5$.

$$
\begin{aligned}
\dot{x}= & {\left[\begin{array}{cc}
-1-\sin ^{2}(2 t) & 2-\frac{1}{2} \sin (4 t) \\
-2-\frac{1}{2} \sin (4 t) & -1-\cos ^{2}(2 t)
\end{array}\right] x } \\
& +\left[\begin{array}{c}
0 \\
1-\rho(t)
\end{array}\right] u
\end{aligned}
$$

where the $\pi$-periodic function $\rho(t)=\sin (2 t)$ for $0 \leq t \leq$ $\frac{\pi}{2}$; otherwise, $\rho(t)=0$. The transition matrix $\phi(t, 0)$ of the example system has a Floquet factorization

$$
\left[\begin{array}{cc}
\cos (2 t) & \sin (2 t) \\
-\sin (2 t) & \cos (2 t)
\end{array}\right] \exp \left\{\left[\begin{array}{cc}
-1 & 0 \\
0 & -2
\end{array}\right] t\right\}
$$

All the assumptions of Theorem 2 are satisfied. For example, the numeric solution for $q=2$ is given by

$$
\begin{aligned}
& {\left[\begin{array}{cc}
0.0000-0.0001 i & -0.0002-0.0000 i \\
-0.0002-0.0000 i & -0.0000+0.00021 i
\end{array}\right] e^{-j 8 t}} \\
& +\left[\begin{array}{cc}
0.0006+0.0006 i & 0.0006-0.0008 i \\
0.0006-0.0008 i & -0.0010-0.0007 i
\end{array}\right] e^{-j 6 t} \\
& +\left[\begin{array}{cc}
0.0603-0.0009 i & 0.0001-0.0600 i \\
0.0001-0.0600 i & -0.0598-0.0006 i
\end{array}\right] e^{-j 4 t} \\
& +\left[\begin{array}{cc}
0.0033+0.0008 i & -0.0013-0.0018 i \\
-0.0013-0.0018 i & 0.0014+0.0043 i
\end{array}\right] e^{-j 2 t} \\
& +\left[\begin{array}{cc}
0.3697+0.0000 i & 0.0024-0.0000 i \\
0.0024-0.0000 i & 0.3662-0.0000 i
\end{array}\right] \\
& +\left[\begin{array}{cc}
0.0603+0.0009 i & 0.0001+0.0600 i \\
0.0001+0.0600 i & -0.0598+0.0006 i
\end{array}\right] e^{j 4 t} \\
& +\left[\begin{array}{cc}
0.0033-0.0008 i & -0.0013+0.0018 i \\
-0.0013+0.0018 i & 0.0014-0.0043 i
\end{array}\right] e^{j 2 t} \\
& +\left[\begin{array}{cc}
0.0000+0.0001 i & -0.0002+0.0000 i \\
-0.0002+0.0000 i & -0.0000-0.0002 i
\end{array}\right] e^{j 8 t} \\
& +\left[\begin{array}{cc}
0.0006-0.0006 i & 0.0006+0.0008 i \\
0.0006+0.0008 i & -0.0010+0.0007 i
\end{array}\right] e^{j 6 t}
\end{aligned}
$$

Fourier coefficients are computed through numerical integrations, and the error tolerance is 0.0001 and the initial value $X_{0,2 q}(t)$ is a zero matrix. We also computed the numeric solutions for the cases $q=1,3,4$. There are no 
significant numeric differences from that in $q=2$. In each case, the algorithm came to the convergent results within 5 rounds of running.

Remark 3. In the above example, the Floquet factorization is given in analytic form for the computations. However, we must point out that the iterative algorithm can still be used even if the Floquet factorization is available only in numeric form. This is because $P(t, 0)$ of the Floquet factorization is needed only in the Fourier coefficient computations, where analytic properties of $P(t, 0)$ have no role to play. The most frequently employed algorithms for numerically computing Floquet factorizations in FDLCP systems are sketched in 23).

\section{Conclusions}

Existence of the so-called harmonic Riccati equations is examined rigorously through an iterative algorithm based on the harmonic Lyapunov theory ${ }^{21)}$ and the contraction mapping theorem. Under some assumptions that are easy to check, the solutions of harmonic Riccati equations are connected with those of periodic matric Riccati equations. This makes it possible to construct an iterative algorithm for solving periodic matric Riccati equations. The study indicates that it is hopeful to extend such results as the $H_{\infty}$ performance of LTI continuous-time systems to FDLCP ones via a class of operator-valued Riccati equations. The details are left for our subsequent study.

\section{References}

1) S. Bittanti, A.J. Laub and J.C. Willems: The Riccati Equation, Springer-Verlag (1991)

2) R.V. Churchill: Fourier Series and Boundary Value Problems, McGraw-Hill, 2nd Ed., 102/105 (1963)

3) M. Farkas: Periodic Motions, Springer-Verlag (1994)

4) H. Fukawa: Mathematics in Control and Vibrations, Corona (1974, in Japanese)

5) I. Gohberg, S. Goldberg and M.A. Kaashoek: Classes of Linear Operators, Birkhäuser, Vol. II (1993)

6) M.O. González: Classical Complex Analysis, Marcel Dekker, Inc. (1992)

7) U.T. Jönsson, C.Y. Kao and A. Megretski: Robustness of periodic trajectories, IEEE Trans. Automat. Contr., 47$11,1842 / 1856$ (2002)

8) H.K. Khalil: Nonlinear Systems, MacMillan (1992)

9) D.L. Kleinman: On an iterative technique for the Riccati equation computations, IEEE Trans. Automat. Contr., 13-1, 114/115 (1968)

10) P. Lancaster and L. Rodman: Algebraic Riccati Equations, Clarendon (1995)

11) D.L. Lukes: Differential Equations: Classical to Controlled, Academic Press (1982)

12) R.D. Milne: Applied Functional Analysis, Pitman Advanced Publishing Program (1980)

13) A.W. Naylor and G.R. Sell: Linear Operator Theory in Engineering and Science, Springer-Verlag (1982)

14) T. Nishimura and H. Kano: Matrix Riccati Equations in
Control Theory, Asakura (1996)

15) R. Ravi, A.M. Pascoal and P.P. Khargonekar: Normalized coprime factorizations for linear time-varying systems, System \& Control Letters, 18, 455/465 (1992)

16) J.A. Richards: Analysis of Periodically Time-Varying Systems, Springer-Verlag (1983)

17) M.A. Shayman: On the phase portrait of the matrix Riccati equation arising from the periodic control problem, SIAM J. Control and Optimization, 23-5, 717/751 (1985)

18) N.M. Wereley: Analysis and Control of Linear Periodically Time Varying Systems, Ph.D. Thesis, Dept. of Aeronautics and Astronautics, M.I.T. (1990)

19) V.A. Yakubovich and V.M. Sturzhinskii: Linear Differential Equations with Periodic Coefficients, John Wiley \& Sons, Vol. I and Vol. II (1975)

20) J. Zhou and T. Hagiwara: Existence conditions and properties of frequency response operators of continuous-time periodic systems, SIAM J. Control and Optimization, 40$6,1867 / 1887(2002)$

21) J. Zhou, T. Hagiwara and M. Araki: Stability analysis of continuous-time periodic systems via the harmonic analysis, IEEE Trans. Automat. Contr., 47-2, 292/298 (2002)

22) J. Zhou: Harmonic Lyapunov equations in continuoustime periodic systems: solutions and properties, IET Control Theory and Applications, 1-4, 946/954 (2007)

23) J. Zhou: Classification and Characteristics of Floquet factorizations in linear continuous-time periodic systems, International Journal of Control (to appear)

24) G.A. Hewer: Periodicity, detectability and the matrix Riccati equation, SIAM J. Control, 13, 1235/1251 (1975)

\section{Appendix A. Proof for Lemma 3}

Clearly, $\left(\mathcal{X},\|\cdot\|_{l_{2} / l_{2}}\right)$ is a normed linear space. To complete the proof, it remains to show that $\left(\mathcal{X},\|\cdot\| l_{l_{2} / l_{2}}\right)$ is complete. To this end, assume that $\left\{\underline{X}_{k}\right\}_{k=0}^{\infty}$ is a Cauchy sequence in $\mathcal{X}$ with $\left\{X_{k}(t)\right\}_{k=0}^{\infty}$ being the defining functions sequence in $L_{\infty}[0, h]$. Then, Corollary 2.2 of 5, p. 567) tells us that $\left\|\underline{X}_{m}-\underline{X}_{n}\right\|_{l_{2} / l_{2}}=$ $\operatorname{ess} \sup _{t \in[0, h]}\left\|X_{m}(t)-X_{n}(t)\right\|$. It follows that $\left\{X_{k}(t)\right\}_{k=0}^{\infty}$ is also a Cauchy sequence in $L_{\infty}[0, h]$. This, together with the fact that $L_{\infty}[0, h]$ is complete (Theorem D.11.4 of 13, p. 590)), implies that there exists $X(t) \in L_{\infty}[0, h]$ such that $\lim _{k \rightarrow \infty} X_{k}(t)=X(t)$ with $\underline{X}=\mathcal{T}\{X(t)\} \in \mathcal{X}$. This, together with Corollary 2.2 of 5 , p. 567), implies that

$$
\begin{aligned}
& \lim _{k \rightarrow \infty}\left\|\underline{X}_{k}-\underline{X}\right\|_{l_{2} / l_{2}} \\
= & \lim _{k \rightarrow \infty} \operatorname{ess} \sup _{t \in[0, h]}\left\|X_{k}(t)-X(t)\right\|=0
\end{aligned}
$$

which says that $\lim _{k \rightarrow \infty} \underline{X}_{k}=\underline{X}$ and thus the completeness of the space $\mathcal{X}$ follows. 


\section{Jun ZHou (Member)}

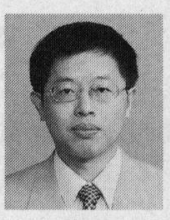

Jun Zhou was born in Lanzhou, Gansu Province, China, in 1963. He received BS degree in Electronics from Sichuan University, and MS degree in Informatics and Control from Lanzhou University in 1984 and 1987, respectively, while he obtained $\mathrm{PhD}$ in Electrical Engineering from Kyoto University in 2002. He has been an associate professor in Dept. of Electrical and Information Engineering, Lanzhou University since 1995. Currently he is with Dept. of Electrical Engineering, Kyoto University. His research interests include system structure algebra, linear periodic systems, nonlinear control systems, harmonics and swing reduction, load flow analysis and voltage stability evaluation in power systems. He is also a member of IEEJ. 